in the BMS group. There was no difference in death, MI or stroke between the stent types. Age-adjusted Cox analysis showed an decrease in the hazard of events for DES compared to BMS (HR $0.60,95 \%$ CIs 0.03 to 0.69 ) and this was maintained with multiple adjustment (HR 0.52, 95\% CIs 0.05 to 0.89 ).

Conclusions In our cohort of patients who had PCI for treatment of SVG disease the use of DES resulted in lower MACE rate compared to BMS over a 5-year follow-up.

\section{DRUG ELUTING STENT IMPLANTATION IS ASSOCIATED WITH LOWER MACE RATES THAN BARE METAL STENT IMPLANTATION IN PRIMARY PCI FOR ST ELEVATION MYOCARDIAL INFARCTION}

doi:10.1136/heartjnl-2012-301877b.38

D I Bromage, ${ }^{*}$ K S Rathod, D A Jones, A Jain, C Knight, A Mathur, A Wragg. Barts and The London NHS Trust, UK

Introduction Drug-eluting stent (DES) implantation during the treatment of ST-elevation myocardial infarction (STEMI) by primary percutaneous coronary intervention (PCI) is controversial due to concerns regarding increased risk of stent thrombosis. We therefore studied long-term outcomes after DES vs bare metal stent (BMS) implantation during primary PCI.

\section{Abstract 038 Table 1}

\begin{tabular}{lllc}
\hline Characteristic & DES $(\mathbf{n = 4 4 2})$ & BMS $(\mathbf{n = 1 7 2 8})$ & p Value \\
\hline Mean age & $46.5 \pm 41.3$ & $53.7 \pm 34.7$ & $<0.0001$ \\
Diabetes & $124(27.3 \%)$ & $272(15.6 \%)$ & $<0.0001$ \\
Previous MI & $76(17.1 \%)$ & $193(11.4 \%)$ & 0.001 \\
Previous CABG & $20(4.4 \%)$ & $35(2.1 \%)$ & 0.001 \\
Previous PCl & $84(18.6 \%)$ & $127(7.5 \%)$ & $<0.0001$ \\
Hypercholesterolaemia & $147(45.4 \%)$ & $498(38.9 \%)$ & 0.033 \\
Multi-vessel disease & $231(62.6 \%)$ & $666(50.1 \%)$ & $<0.0001$ \\
Mean stent length $(\mathrm{mm})$ & $22.3 \pm 5.1$ & $20.1 \pm 5.4$ & $<0.0001$ \\
Mean stent width $(\mathrm{mm})$ & $3.4 \pm 0.4$ & $31.9 \pm 0.9$ & $<0.0001$ \\
\hline
\end{tabular}

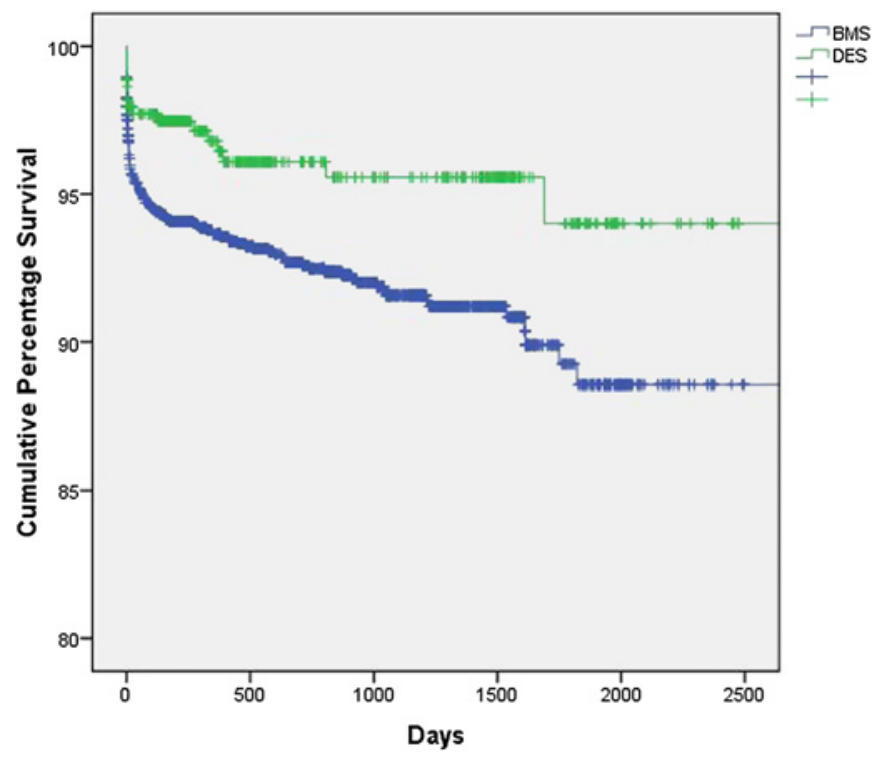

Abstract 038 Figure 1 MACE in DES vs BMS implantation in primary $\mathrm{PCl}$.
Methods Between October 2003 and September 2010, 2170 consecutive patients undergoing primary PCI for STEMI at a single high-volume London centre were included. The primary end point was major adverse cardiac events (MACE), defined as death, myocardial infarction (MI), stroke and target vessel revascularisation (TVR). Median follow-up was 2 years (IOR 0.7-3.6 years).

Results 442 patients underwent PCI with DES and 1728 with BMS. Differences in baseline characteristics are outlined in Abstract 038 table 1. There was a significant difference in MACE between the groups in favour of DES (3.9\% vs $7.7 \%, p=0.006$ ) (Abstract 038 figure 1). Age-adjusted Cox analysis demonstrated the decrease in MACE in patients undergoing DES compared to BMS implantation was maintained with multiple adjustment (HR 0.60 [95\% CI 0.37 to 0.96]). In addition, after regression adjustment incorporating a propensity score (age, stent length, stent width, gender, ethnicity, previous MI, PCI or coronary artery bypass grafting (CABG), diabetes, hypertension, hypercholesterolaemia, smoking status, presence or absence of shock, and ejection fraction) into the hazards model as a covariate, this difference persisted (HR 0.61 [95\% CI 0.37 to 0.96$])$.

Conclusion DES implantation is associated with lower MACE rates than BMS implantation in primary PCI for STEMI.

\section{OUTCOMES FOLLOWING UNPROTECTED LEFT MAIN STENTING WITH FIRST VS SECOND GENERATION DRUG- ELUTING STENTS: THE MILAN EXPERIENCE}

doi:10.1136/heartjnl-2012-301877b.39

G L Buchanan, ${ }^{*}$ C Bernelli, A lelasi, M Montorfano, A Latib, F Figini, M Slavich, F M Sacco, I Franzoni, M Carlino, S Ferrarello, A Colombo. San Raffaele Scientific Institute, Italy

Background Second-generation drug-eluting stents (DES) are said to be more safe and effective. Our aim was to assess clinical outcomes following first- vs second-generation DES implantation in patients undergoing unprotected left main (ULMCA) percutaneous coronary intervention.

Methods All consecutive patients from our single-center prospective registry treated for ULMCA with DES implantation from January 2005 to November 2010 were analysed. The study endpoint was major adverse cardiac event (MACE) defined as all-cause mortality, target lesion revascularisation (TLR), and target vessel revascularisation (TVR) at clinical follow-up.

Results A total of 179 patients were included: mean age $66.5 \pm 12.7$ years and $83.2 \%$ were male with mean left ventricular ejection fraction $54.6 \pm 8.4 \%$ and SYNTAX score $23.3 \pm 31.2$. The median follow-up was 705.5 days (IOR 339.8-1168.0). First-generation DES were used in $53.1 \%$ (of which $51.6 \%$ were sirolimus and paclitaxel $48.4 \%$ ) and $46.9 \%$ had second-generation DES $(85.7 \%$ everolimus; $11.9 \%$ zotorolimus; $2.4 \%$ biolimus). Interestingly, there were more patients with diabetes treated with first-generation ( $30.5 \%$ vs $14.3 \% ; p=0.023$ ). Regarding the procedure, intravascular ultrasound guidance was similar between first and second-generation (respectively $47.4 \%$ vs $59.5 \%$; $=0.130$ ). There were more patients in the first-generation group with distal ULMCA disease (82.1\% vs $67.9 \% ; p=0.064)$. At follow-up, there was a significant difference in MACE favouring second-generation (30.5\% vs $19.0 \%$ $\mathrm{p}=0.047)$, most related to a reduction in the TLR $(13.7 \%$ vs $4.8 \%$; $p=0.026)$ and TVR $(24.2 \%$ vs $14.3 \% ; p=0.031)$. However, there was no difference in all-cause mortality $(10.5 \%$ vs $7.1 \%$; $=0.138)$ with a trend for increased cardiovascular mortality in those treated by firstgeneration ( $8.4 \%$ vs $2.4 \% ; \mathrm{p}=0.082$ ). Moreover, there was no difference in definite/probable stent thromboses $(5.3 \%$ vs $2.4 \%$; $\mathrm{p}=0.114$ ).

Conclusions Second-generation DES have improved results with regards to MACE at mid-term follow-up, perhaps secondary to patient selection. This needs to be confirmed at longer-term follow-up. 